\title{
Global Existence, Uniqueness of Weak Solutions and Determining Functionals for Nonlinear Wave Equations
}

\author{
Ülkü Dinlemez \\ Department of Mathematics, Gazi University, Ankara, Turkey \\ Email: ulku@gazi.edu.tr
}

Received April 21, 2013; revised May 30, 2013; accepted July 3, 2013

Copyright (C) 2013 Ülkü Dinlemez. This is an open access article distributed under the Creative Commons Attribution License, which permits unrestricted use, distribution, and reproduction in any medium, provided the original work is properly cited.

\begin{abstract}
We consider the initial-boundary value problem for a nonlinear wave equation with strong structural damping and nonlinear source terms in IR. We prove the global existence and uniqueness of weak solutions of the problem and then we will study the determining modes on the phase space $H^{2}(0,1) \cap H_{0}^{1}(0,1)$ by using energy methods and the concept of the completeness defect.
\end{abstract}

Keywords: Global Existence; Uniqueness; Determining Modes

\section{Introduction}

In this paper we study the initial-boundary value problem for the following nonlinear wave equation

$$
\begin{gathered}
u_{t t}-\alpha u_{x x}-\alpha u_{t x x}+|u|^{2} u-\mu\left(\left|u_{x}\right|^{2} u_{x}\right)_{x}=f(x), \\
x \in(0,1), t \in[0, \infty)
\end{gathered}
$$

with boundary conditions

$$
u(0, t)=u(1, t)=0, t>0
$$

and initial conditions

$$
u(x, 0)=u_{0}(x), u_{t}(x, 0)=u_{1}(x), x \in(0,1)
$$

where $\alpha>1$ constant, $u_{t x x}$ is a strong structural damping term, $|u|^{2} u$ is nonlinear source term and $\left(\left|u_{x}\right|^{2} u_{x}\right)_{x}$ is a nonlinear strain term.

An other version of problems (1.1)-(1.3) was studied in [1-4]. In [1] Chen et al worked that the following initial boundary value problem

$$
\begin{gathered}
u_{t t}=\alpha u_{t x x}+\sigma\left(u_{x}\right)_{x}-f(u)+g(x), \\
x \in(0,1), t \in[0, \infty) \\
u(0)=u_{0}(x), u_{t}(0)=u_{1}(x), x \in(0,1) \\
u(0, t)=u(1, t)=0, t>0
\end{gathered}
$$

has a global solution and there exists a compact global attractor with finite dimension. In [2] Karachalios and Staurakalis studied the local existence for (1.1) with $\mu=0, \quad u_{t}$ is a damping term and without nonlinear source term. In [3] Çelebi and Uğurlu gave the existence of a wide collection of finite sets of functionals on the phase space $H^{2}(0,1) \cap H_{0}^{1}(0,1)$ that completely determines asymptotic behavior of solutions to the strongly damped nonlinear wave equations. In [4] Chueshov presented the approach of a set of determining functionals containing determining modes and nodes that completely determines the long-time behavior of some first and second order evolution equations.

Similar results of determining modes for similar equations have been obtained in [5-7].

In this article, we take the problem defined by (1.1)(1.3) which was not investigated in above mentioned articles. Our problem has nonlinear strain and source terms. The control of long time behavior is achieved due to the presence of restoring forces $\left(\left|u_{x}\right|^{2} u_{x}\right)_{x}$. In Section 2 under conditions

$\left(u_{0}, u_{1}\right) \in H^{2}(0,1) \cap H_{0}^{1}(0,1) \times L^{2}(0,1), \quad f(x) \in L^{2}(0,1)$ and $\alpha>1$, we prove the global existence and uniqueness of a weak solution $u$ of the problems (1.1)-(1.3). In Section 3 we study determining modes on the phase space $H^{2}(0,1) \cap H_{0}^{1}(0,1)$ by using energy methods and the concept of the completeness defect. 


\section{The Global Existence and Uniqueness of Weak Solutions}

Let $L^{2}(0,1)$ be the usual Hilbert space of square integrable functions with the standard $L^{2}$ norm $\|$.$\| and$ inner product (.,.). Denote $A=-\frac{\partial^{2}}{\partial x^{2}}$, the Laplacian operator on $L^{2}$ with domain $D(A)=H^{2}(0,1) \cap H_{0}^{1}(0,1)$. $A$ is a sectorial operator and that $A^{-1}$ is a bounded linear operator defined in $L^{2}(0,1)$ see [8]. The nonlinear source term $g(u)=|u|^{2} u$ satisfies the following conditions

$$
\lim _{|s| \rightarrow \infty} \inf \frac{G(s)}{s^{2}} \geq 0,
$$

there exists a constant $0<w$ such that

$$
\lim _{|s| \rightarrow \infty} \inf \frac{s g(s)-w G(s)}{s^{2}} \geq 0,
$$

where $G(s)=\int_{0}^{s} g(t) \mathrm{d} t$. Finally we denote $Y=D(A) \times L^{2}(0,1)$ with the standard product norm

$$
\begin{gathered}
\|(u, v)\|_{Y}=\left(\|A u\|^{2}+\|v\|^{2}\right)^{\frac{1}{2}} \text {. Define } A_{\alpha} \text { in } Y \text { by } \\
A_{\alpha}(u, v)=(v,-\alpha A v-\alpha A u), \\
(u, v) \in D\left(A_{\alpha}\right)=D(A) \times D(A) .
\end{gathered}
$$

Then the following Lemma1 is valid [9].

Lemma $1 A_{\alpha}$ is a sectorial operator on $Y$.

We define a map $G_{\alpha}$ from $D\left(A_{\alpha}\right)$ to $Y$ by

$$
\begin{gathered}
G_{\alpha}(u, v)=\left(0,-|u|^{2} u+3 \mu\left|u_{x}\right|^{2} u_{x x}+f(x)\right), \\
\text { for }(u, v) \in D\left(A_{\alpha}\right)
\end{gathered}
$$

where $f \in L^{2}(0,1)$.

Using the Sobolev embedding theorem, we can see that $G_{\alpha}$ is locally Lipschitz continuous. Thus we apply the existence theorem in [8] to get the solutions of initial value problem for the following system in $Y$ :

$$
Z_{t}=A_{\alpha} Z+G_{\alpha} Z, Z=(u, v) \in Y
$$

when

$$
\begin{aligned}
& u_{t}=v \\
& v_{t}=\alpha v_{x x}+\alpha u_{x x}-|u|^{2} u+3 \mu\left|u_{x}\right|^{2} u_{x x}+f(x) .
\end{aligned}
$$

Now, we have the following theorem.

Theorem 2 (Local existence) For $\left(u_{0}, u_{1}\right) \in Y$ and $0<w<1$, there exists $t_{0}=t_{0}\left(u_{0}, u_{1}\right)>0$ such that $\left(u, u_{t}\right) \in C\left(\left[0, t_{0}\right) ; Y\right), \quad u_{t t} \in L^{2}(0,1)$ and $u_{t x x} \in L^{2}(0,1)$ for a.e. $t \in\left(0, t_{0}\right)$ and $u$ satisfies (1.1)-(1.3). Moreover, if $t_{0}$ is maximal, then either $t_{0}=\infty$ or $\|(u, v)\|_{Y}$ is unbounded on $\left[0, t_{0}\right)$.

Now for the proof of the Theorem 4 (Global Existence) we give the following Lemma 3. In the proofs of Lemma 3 and Theorem 4 (Global Existence) we repeat a similar technique used in [1].

Lemma 3 For $\left(u_{0}, u_{1}\right) \in Y$ and $0<w<1$, there exist constants $\rho=\rho(\alpha, w), \quad \Psi=\Psi\left(\left\|u_{0}\right\|^{2},\left\|u_{1}\right\|^{2}, K\right)$,

$$
\Phi=\Phi\left(K, w,\|f\|^{2}\right) \text { such that for } t \in\left(0, t_{0}\right)
$$$$
\left\|u_{t}\right\|^{2}+\left\|u_{x}\right\|^{2}+\|u\|^{2} \leq \Psi \mathrm{e}^{-w \rho t}+\Phi
$$

where $u$ is the solution of (1.1)-(1.3).

Proof. Let $v=u_{t}+\rho u$ where $\rho$ is a constant to be determined. Thus (1.1) becomes

$$
\begin{aligned}
& v_{t}-\alpha v_{x x}-\rho v+\rho^{2} u+\alpha(\rho-1) u_{x x} \\
& +|u|^{2} u-\mu\left(\left|u_{x}\right|^{2} u_{x}\right)_{x}=f(x)
\end{aligned}
$$

Taking the inner product of both sides of (2.6) with $v$ and integrating the resulting equation, we have

$$
\frac{\mathrm{d}}{\mathrm{d} t} A_{1}(u, v)+B_{1}(u, v)=0
$$

where

$$
\begin{aligned}
A_{1}(u, v)= & \frac{1}{2}\|v\|^{2}+\frac{\rho^{2}}{2}\|u\|^{2}-\frac{\alpha(\rho-1)}{2}\left\|u_{x}\right\|^{2} \\
& +\frac{1}{4}\|u\|_{4}^{4}+\frac{\mu}{4}\left\|u_{x}\right\|_{4}^{4}-(f, u),
\end{aligned}
$$

and

$$
\begin{aligned}
B_{1}(u, v)= & \alpha\left\|v_{x}\right\|^{2}-\rho\|v\|^{2}+\rho^{3}\|u\|^{2}-\rho \alpha(\rho-1)\left\|u_{x}\right\|^{2} \\
& +\rho\|u\|_{4}^{4}+\mu \rho\left\|u_{x}\right\|_{4}^{4}-\rho(f, u) .
\end{aligned}
$$

Now we will estimate $A_{1}(u, v)$ and $B_{1}(u, v)$. Choose $\rho$ such that

$$
\rho<\min \left\{\frac{\alpha \lambda_{1}}{1+\frac{w}{2}}, \frac{\lambda_{1}}{1+\lambda_{1}}, \frac{\lambda_{1} \alpha}{\lambda_{1} \alpha+2(1-w)^{2}}\right\}
$$

where $\lambda_{1}>0$ is first eigenvalue of the following problem

$$
\begin{gathered}
\Delta u+\lambda u=0 \text { in }(0,1) \\
u(0)=u(1)=0 .
\end{gathered}
$$

From (2.8) and (2.9) with $0<w<1$, we get

$$
\begin{aligned}
& B_{1}(u, v)-\rho w A_{1}(u, v) \\
& \geq\left(\alpha \lambda_{1}-\rho\left(1+\frac{w}{2}\right)\right)\|v\|^{2}+\rho \alpha(\rho-1)\left(\frac{w}{2}-1\right)\left\|u_{x}\right\|^{2} \\
& \quad+\rho^{3}\left(1-\frac{w}{2}\right)\|u\|^{2}+\rho\left(1-\frac{w}{4}\right)\|u\|_{4}^{4} \\
& \quad+\mu \rho\left(1-\frac{\mu}{4}\right)\left\|u_{x}\right\|_{4}^{4}-\rho(1-w)(f, u) .
\end{aligned}
$$


We use Young inequality, Poincaré inequality and (2.10) in (2.11) we find

$$
B_{1}(u, v)-\rho w A_{1}(u, v) \geq-\frac{1}{4}\|f\|^{2} .
$$

Similarly, we obtain

$$
\begin{aligned}
A_{1}(u, v) \geq & \frac{1}{2}\|v\|^{2}+\frac{\rho^{2}}{2}\|u\|^{2} \\
& +\frac{\alpha}{2}\left(1-\rho\left(1+\frac{1}{\lambda_{1}}\right)\right)\left\|u_{x}\right\|^{2}-\frac{1}{\rho \alpha}\|f\|^{2} .
\end{aligned}
$$

Then (2.7), (2.12) and (2.13) yield

$$
\frac{\mathrm{d}}{\mathrm{d} t} A_{1}(u, v)+\rho w A_{1}(u, v) \leq \frac{1}{4}\|f\|^{2} .
$$

Using Gronwall's inequality, we have

$$
A_{1}(u, v) \leq A_{1}\left(u_{0}, u_{1}\right) \mathrm{e}^{-\rho w t}+\frac{1}{4 \rho w}\|f\|^{2}\left(1-\mathrm{e}^{-\rho w t}\right)
$$

Since $u_{0} \in H^{2}(0,1) \cap H_{0}^{1}(0,1)$, we can find that $\left|A_{1}\left(u_{0}, u_{1}\right)\right| \leq C\left(\left\|u_{0}\right\|^{2},\left\|u_{1}\right\|^{2}, \rho\right)$ by using the Sobolev embedding theorem. Thus using (2.13) in (2.15) we obtain

$$
\begin{aligned}
& \frac{1}{2}\|v\|^{2}+\frac{\rho^{2}}{2}\|u\|^{2}+\frac{\alpha}{2}\left(1-\rho\left(1+\frac{1}{\lambda_{1}}\right)\right)\left\|u_{x}\right\|^{2} \\
& \leq C \mathrm{e}^{-\rho w t}+\frac{1}{2 \rho}\left(\frac{1}{2 w}+\frac{1}{\alpha}\right)\|f\|^{2} .
\end{aligned}
$$

Taking

$$
K=\min \left\{\frac{1}{2}, \frac{\rho^{2}}{2}, \frac{\alpha}{2}\left(1-\rho\left(1+\frac{1}{\lambda_{1}}\right)\right)\right\}
$$

and choosing $\rho$ we get (2.5).

Now we can prove the global existence of the problems (1.1)-(1.3).

Theorem 4 (Global Existence) For $\left(u_{0}, u_{1}\right) \in Y$, there exists a global solution $u$ of problems (1.1)-(1.3) satisfying $\left(u, u_{t}\right) \in C([0, \infty) ; Y)$.

Proof. In Theorem 2 (Local Existence) we know that $\left(u, u_{t}\right) \in Y$ for $t \in\left[0, t_{0}\right)$ and $u_{t t} \in L^{2}(0,1), u_{t x x} \in L^{2}(0,1)$ for a.e. $t \in\left(0, t_{0}\right)$. In Lemma 3 we find that $\left\|u_{x}\right\|$, $\left\|u_{t}\right\|$ and $\|u\|$ are uniformly bounded for all $t>0$. Now we prove the global existence of the solution $u$. To do this we need to show that $\left\|u_{x x}\right\|$ is uniformly bounded for $t>0$.

Now, taking the inner product of both sides (1.1) in $L^{2}(0,1)$ with $-u_{x x}$, we have

$$
\begin{aligned}
& \frac{\mathrm{d}}{\mathrm{d} t}\left\{\frac{\alpha}{2} \|\left. u_{x x}\right|^{2}-\left(u_{t}, u_{x x}\right)\right\}-\left\|u_{t x}\right\|^{2}+3 \int_{0}^{1}|u|^{2}\left|u_{x}\right|^{2} \mathrm{~d} x \\
& +3 \mu \int_{0}^{1}\left|u_{x}\right|^{2}\left|u_{x x}\right|^{2} \mathrm{~d} x+\alpha\left\|u_{x x}\right\|^{2}+\left(f, u_{x x}\right)=0 .
\end{aligned}
$$

Then we multiply both sides of (2.17) by $\alpha$ and add to $(2.7)$ to obtain

$$
\begin{aligned}
& \frac{\mathrm{d}}{\mathrm{d} t}\left\{\frac{\alpha^{2}}{2}\left\|u_{x x}\right\|^{2}-\alpha\left(u_{t}, u_{x x}\right)+A_{1}(u, v)\right\} \\
& +\alpha^{2}\left\|u_{x x}\right\|^{2}-\alpha\left\|u_{t x}\right\|^{2}+\alpha\left(f, u_{x x}\right)+3 \alpha \int_{0}^{1}|u|^{2}\left|u_{x}\right|^{2} \mathrm{~d} x \\
& +3 \mu \alpha \int_{0}^{1}\left|u_{x}\right|^{2}\left|u_{x x}\right|^{2} \mathrm{~d} x+\alpha\left\|v_{x}\right\|^{2}-\rho\|v\|^{2}+\rho^{3}\|u\|^{2} \\
& -\rho \alpha(\rho-1)\left\|u_{x}\right\|^{2}+\rho\|u\|_{4}^{4}+\mu \rho\left\|u_{x}\right\|_{4}^{4}-\rho(f, u)=0 .
\end{aligned}
$$

Using Poincaré inequality and (2.10) in (2.18), we have

$$
\frac{\mathrm{d}}{\mathrm{d} t} A_{2}\left(u, u_{t}\right)+B_{2}\left(u, u_{t}\right) \leq 0
$$

where

$$
A_{2}\left(u, u_{t}\right)=\frac{\alpha^{2}}{2}\left\|u_{x x}\right\|^{2}-\alpha\left(u_{t}, u_{x x}\right)+A_{1}(u, v)
$$

and

$$
\begin{aligned}
B_{2}\left(u, u_{t}\right)= & \alpha^{2}\left\|u_{x x}\right\|^{2}+\alpha\left(f, u_{x x}\right)+3 \alpha \int_{0}^{1}|u|^{2}\left|u_{x}\right|^{2} \mathrm{~d} x \\
& +3 \mu \alpha \int_{0}^{1}\left|u_{x}\right|^{2}\left|u_{x x}\right|^{2} \mathrm{~d} x-\rho\left\|u_{t}\right\|^{2} \\
& -\alpha \rho\left\|u_{x}\right\|^{2}-\rho \alpha(\rho-1)\left\|u_{x}\right\|^{2} \rho^{3}\|u\|^{2} \\
& +\rho\|u\|_{4}^{4}+\mu \rho\left\|u_{x}\right\|_{4}^{4}-\rho(f, u) .
\end{aligned}
$$

Then thanks to Young inequality we obtain

$$
\begin{aligned}
& B_{2}\left(u, u_{t}\right)-\rho A_{2}\left(u, u_{t}\right) \\
& \geq-\|f\|^{2}-\alpha \rho\left\|u_{x}\right\|^{2}-3 \rho\left\|u_{t}\right\|^{2}-\frac{\rho^{3}}{2}\|u\|^{2} .
\end{aligned}
$$

Taking $C_{3}=\max \left\{1, \rho \alpha, 3 \rho, \frac{\rho^{3}}{2}\right\}$ in (2.22) we get

$$
\begin{aligned}
& B_{2}\left(u, u_{t}\right)-\rho A_{2}\left(u, u_{t}\right) \\
& \geq-C_{3}\left\{\|f\|^{2}+\left\|u_{x}\right\|^{2}+\left\|u_{t}\right\|^{2}+\|u\|^{2}\right\} .
\end{aligned}
$$

Using (2.19), (2.23) and Gronwall's inequality we get

$$
\begin{aligned}
& A_{2}\left(u, u_{t}\right) \leq A_{2}\left(u(0), u_{t}(0)\right) \mathrm{e}^{-\rho t} \\
& +C_{3} \int_{0}^{t} \mathrm{e}^{-\rho(t-s)}\left\{\|f\|^{2}+\left\|u_{x}\right\|^{2}+\left\|u_{t}\right\|^{2}+\|u\|^{2}\right\} \mathrm{d} s .
\end{aligned}
$$

Thus (2.24) and Lemma 3 imply that $A_{2}\left(u, u_{t}\right)$ is uniformly bounded in $[0, \infty)$, because of $A_{2}\left(u(0), u_{t}(0)\right) \leq C_{4}$, for some constant $C_{4}=C_{4}\left(\left\|u(0), u_{t}(0)\right\|_{Y}\right)$ and we have

$$
\begin{aligned}
& A_{2}\left(u, u_{t}\right) \\
& \geq \frac{\alpha^{2}}{4}\left\|u_{x x}\right\|^{2}-C_{5}\left\{\|f\|^{2}+\left\|u_{x}\right\|^{2}+\left\|u_{t}\right\|^{2}+\|u\|^{2}\right\}
\end{aligned}
$$


where $C_{5}=\max \left\{1, \frac{1}{4 \rho^{2}}\right\}$. Finally, using Sobolev embedding theorem and Lemma 3 we obtain that $\left\|u_{x x}\right\|$ is uniformly bounded in $[0, \infty)$.

Theorem 5 (Uniqueness of weak solution) $A$ weak solution of (1.1)-(1.3) is unique.

Proof. Let $u$ and $v$ be two distinct solutions to (1.1)(1.3) for the same initial and boundary data. We define the difference of these solutions as $w=u-v$. Then from (1.1)-(1.3), $w$ satisfies

$$
\begin{gathered}
w_{t t}-\alpha w_{x x}-\alpha w_{t x x}+\left(|u|^{2} u-|v|^{2} v\right) \\
-\mu\left(\left(\left|u_{x}\right|^{2} u_{x}\right)_{x}-\left(\left|v_{x}\right|^{2} v_{x}\right)_{x}\right)=0, \\
x \in(0,1), t \in[0, \infty) \\
w(0, t)=w(1, t)=0, t>0 \\
w(x, 0)=0, w_{t}(x, 0)=0, x \in(0,1)
\end{gathered}
$$

Taking the inner product of $(2.26)$ by $w_{t}$ in $L^{2}(0,1)$ and integrating by parts gives

$$
\begin{aligned}
& \frac{1}{2} \frac{\mathrm{d}}{\mathrm{d} t}\left\{\left\|w_{t}||^{2}+\alpha\right\| w_{x} \|^{2}\right\}+\alpha\left\|w_{t x}\right\|^{2} \\
& \leq\left.\mu \int_{0}^{1}|| u_{x}\right|^{2} u_{x}-\left|v_{x}\right|^{2} v_{x}|| w_{t x} \mid \mathrm{d} x \\
& +\left.\int_{0}^{1}|| u\right|^{2} u-|v|^{2} v|| w_{t} \mid \mathrm{d} x .
\end{aligned}
$$

By means of the inequality

$$
\left.|| x\right|^{\tau-2} x-|y|^{\tau-2} y\left|\leq(\tau-1) R^{\tau-2}\right| x-y \mid,
$$

which holds for all $x, y \in[-R, R], \quad R>0$ and $\tau \geq 2$, it follows from (2.29) that

$$
\begin{aligned}
& \frac{\mathrm{d}}{\mathrm{d} t}\left\{\left\|w_{t}\right\|^{2}+\alpha\left\|w_{x}\right\|^{2}\right\}+\alpha\left\|w_{t x}\right\|^{2} \\
& \leq\left(\frac{3 R_{1}^{2}}{\lambda_{1}}+\frac{9 \mu^{2} R_{2}^{4}}{\alpha}\right)\left\|w_{x}\right\|^{2}+3 R_{1}^{2}\left\|w_{t}\right\|^{2} .
\end{aligned}
$$

Thus we get

$$
\frac{\mathrm{d}}{\mathrm{d} t}\left\{\left\|w_{t}\right\|^{2}+\alpha\left\|w_{x}\right\|^{2}\right\}+\alpha\left\|w_{t x}\right\|^{2} \leq \hbar\left\{\left\|w_{x}\right\|^{2}+\left\|w_{t}\right\|^{2}\right\}
$$

where $\hbar=\max \left\{\frac{3 R_{1}^{2}}{\lambda_{1}}+\frac{9 \mu^{2} R_{2}^{4}}{\alpha}, 3 R_{1}^{2}\right\}$. Consequently the differential form of Gronwall's inequality implies to give $w(t)=0$ on $[0, T]$.

\section{Existence of Determining Functionals}

Now we give some definitions, theorems and corollary for proving existence of determining functionals.
Definition 6 [4] Let $\mathfrak{I}=\left\{\ell_{j}: j=1, \cdots, n\right\}$ be a finite set of linear continuous functionals on $H^{2}(0,1) \cap H_{0}^{1}(0,1)$. We will say that $\mathfrak{I}$ is a set of determining functionals for (1.1)-(1.3) when for any two solutions $u, v \in C\left([0, \infty), H^{2}(0,1) \cap H_{0}^{1}(0,1)\right)$ with $u_{t}$ and $v_{t} \in C\left([0, \infty), L^{2}(0,1)\right)$, the conditions

$$
\lim _{t \rightarrow \infty} \int_{t}^{t+1}\left|\ell_{j}(u(k)-v(k))\right|^{2} \mathrm{~d} k=0, j=1,2, \cdots, n
$$

imply

$$
\lim _{t \rightarrow \infty}\left[\left\|u_{x x}(t)-v_{x x}(t)\right\|^{2}+\left\|u_{t}(t)-v_{t}(t)\right\|^{2}\right]=0 .
$$

Definition 7 [4] Let $V$ and $H$ be the reflexive Banach spaces and $V$ be continuously and densely embedded into $H$. Let $\mathfrak{I}=\left\{\ell_{j}: j=1, \cdots, n\right\}$ be a set of linear functionals on $V$. We define the completeness defect

$\beta_{\mathfrak{I}}=\beta_{\mathfrak{I}}(V, H)$ of the set $\mathfrak{I}$ with respect to the pair of the spaces $V$ and $H$ by the formula

$$
\begin{aligned}
& \beta_{\mathfrak{I}}(V, H) \\
& =\sup \left\{\|u\|_{H}: u \in V, \ell_{j}(u)=0, \ell_{j} \in \mathfrak{I},\|u\|_{V} \leq 1\right\} .
\end{aligned}
$$

The following assertion gives the spectral characterization of the completeness defect in the case when $V$ and $H$ are the Hilbert spaces.

Theorem 8 [4] Let $V$ and $H$ be the separable Hilbert spaces such that $V$ is compactly and densely embedded into $H$. Let $K$ be the self-adjoint, positive and compact operator in the space $V$ defined by the equality

$$
(K u, v)_{V}=(u, v)_{H}
$$

for $u, v \in V$. Then the completeness defect $\beta_{\mathfrak{I}}(V, H)$ of a set $\mathfrak{I}$ of linear functionals on $V$ can be evaluated by the formula

$$
\beta_{\mathfrak{\Im}}(V, H)=\sqrt{\gamma_{\max }\left(P_{\mathfrak{I}} K P_{\mathfrak{I}}\right)}
$$

where $P_{\mathfrak{\Im}}$ is the orthoprojector in the space $V$ on the annihilator

$$
\mathfrak{I}^{\perp}=\{u \in V: \ell(v)=0, \ell \in \mathfrak{I}\},
$$

$\gamma_{\max }(S)$ is the maximal eigenvalue of the operator $S$.

Corollary 9 [4] Let the conditions of Theorem 8 be hold and let us denote by $\left\{e_{i}\right\}$ the orthonormal basis in the space $V$ that consists of the eigenvectors of the operator $K$ :

$$
\begin{aligned}
& K e_{i}=\gamma_{i} e_{i},\left(e_{i}, e_{j}\right)_{V}=\delta_{i j}, \\
& \gamma_{1} \geq \gamma_{2} \geq \cdots, \lim _{n \rightarrow \infty} \gamma_{n}=0 .
\end{aligned}
$$

Then the completeness defect of the set of functionals,

$$
\mathfrak{I}=\left\{\ell_{j} \in V^{*}: \ell_{j}(u)=\left(u, e_{j}\right)_{V}, j=1,2, \cdots, n\right\}
$$


can be evaluated by the formula

$$
\beta_{\mathfrak{I}}(V, H)=\sqrt{\gamma_{n+1}} .
$$

The following theorem establishes a relation between the completeness defect and the set $\mathfrak{I}$.

Theorem 10 [4] Let $\beta_{\mathfrak{I}}=\beta_{\mathfrak{I}}(V, H)$ be the completeness defect of a set $\mathfrak{I}$ of linear functionals on $V$ with respect to $H$. Then there exists a positive constant $C_{\mathfrak{I}}$ such that

$$
\|u\|_{H} \leq C_{\mathfrak{J}} \sup \left\{|\ell(u)|: \ell \in \mathfrak{I},\|\ell\|_{*} \leq 1\right\}+\beta_{\mathfrak{I}}\|u\|_{V}
$$

for any $u \in V$, where $\mathfrak{I}$ is the closed linear span of the set $\mathfrak{I}$ in $V^{*}$, the dual space of $V$ and $\|\cdot\|_{*}$ is the norm in $V^{*}$.

The following version of Gronwall's lemma is also needed to determine behavior of solutions as $t \rightarrow \infty$.

Lemma 11 [4] Let $\zeta$ be a locally integrable real valued function on $(0, \infty)$, satisfying for some $0<T<\infty$ the following conditions

$$
\begin{gathered}
\liminf _{t \rightarrow \infty} \int_{t}^{t+T} \zeta(z) \mathrm{d} z=\theta>0, \\
\limsup _{t \rightarrow \infty} \int_{t}^{t+T} \zeta^{-}(z) \mathrm{d} z=\Gamma<\infty,
\end{gathered}
$$

where $\zeta^{-}=\max \{-\zeta, 0\}$. Further, let $\kappa$ be a real valued locally integrable function defined on $(0, \infty)$ such that

$$
\lim _{t \rightarrow \infty} \int_{t}^{t+T} \kappa^{+}(z) \mathrm{d} z=0,
$$

where $\kappa^{+}=\max \{\kappa, 0\}$. Suppose that $\xi$ is an absolutely continuous non-negative function on $(0, \infty)$ such that

$$
\frac{\mathrm{d} \xi}{\mathrm{d} t}+\zeta \xi \leq \kappa, \text { a.e. on }(0, \infty) .
$$

Then $\xi(t) \rightarrow 0$ as $t \rightarrow \infty$.

Now we can prove the main result concerning existence of a set of determining functionals of solutions to problems (1.1)-(1.3).

Theorem 12 Let $\mathfrak{I}=\left\{\ell_{j}: j=1, \cdots, n\right\}$ be a set of linear continuous functionals on the space $H^{2}(0,1) \cap$ $H_{0}^{1}(0,1)$ and let $\beta_{\mathfrak{I}}=\beta_{\mathfrak{I}}\left(H^{2}(0,1) \cap H_{0}^{1}(0,1), L^{2}(0,1)\right)$ be a positive number satisfying

$A=\frac{\alpha}{2}-2\left(9 R_{5}^{4}+18 R_{3}^{4}\right) \beta_{\Im}^{2}>0$ where $\alpha>1, R_{3}, R_{5}$ positive constants. Then, $\mathfrak{I}$ is a set of determining functionals for (1.1)-(1.3).

Proof. Let $u$ and $v$ be two solutions of problems (1.1)(1.3). Let $w=u-v$ be the difference of these solutions. Thus $w$ satisfies (2.26)-(2.28). Now taking the $L^{2}(0,1)$ inner product of (2.26) by $w_{t}$ we get

$$
\begin{aligned}
& \frac{1}{2} \frac{\mathrm{d}}{\mathrm{d} t}\left\{\left\|w_{t}\right\|^{2}+\alpha\left\|w_{x}\right\|^{2}\right\}+\alpha\left\|w_{t x}\right\|^{2} \\
& =-\mu \int_{0}^{1}\left(\left(\left|u_{x}\right|^{2} u_{x}\right)_{x}-\left(\left|v_{x}\right|^{2} v_{x}\right)_{x}\right) w_{t x} \mathrm{~d} x \\
& \quad-\int_{0}^{1}\left(|u|^{2} u-|v|^{2} v\right) w_{t} \mathrm{~d} x .
\end{aligned}
$$

Using (2.30) and Young inequality in right hand side of (3.10) we obtain

$$
\begin{aligned}
& \frac{\mathrm{d}}{\mathrm{d} t}\left\{\left\|w_{t}\right\|^{2}+\alpha\left\|w_{x}\right\|^{2}\right\}+2 \alpha\left\|w_{t x}\right\|^{2} \\
& \leq 18 R_{3}^{4}\|w\|^{2}+\frac{1}{2}\left\|w_{t}\right\|^{2}+\frac{1}{2}\left\|w_{x}\right\|^{2}+18 \mu^{2} R_{2}^{4}\left\|w_{t x}\right\|^{2} .
\end{aligned}
$$

On the other hand, the $L^{2}$ inner product of (2.26) by $-w_{x x}$ and integration by parts over $(0,1)$ yields

$$
\begin{aligned}
& \frac{\mathrm{d}}{\mathrm{d} t}\left\{\frac{\alpha}{2}\left\|w_{x x}\right\|^{2}-\left(w_{t}, w_{x x}\right)\right\}-\left\|w_{t x}\right\|^{2}+\alpha\left\|w_{x x}\right\|^{2} \\
& =\int_{0}^{1}\left(|u|^{2} u-|v|^{2} v\right) w_{x x} \mathrm{~d} x \\
& \quad-\mu \int_{0}^{1}\left(\left(\left|u_{x}\right|^{2} u_{x}\right)_{x}-\left(\left|v_{x}\right|^{2} v_{x}\right)_{x}\right) w_{x x} \mathrm{~d} x .
\end{aligned}
$$

We assume that for some $p \geq 2$ and any small $v, v_{1}$, $v_{2} \in \mathbb{R}$, the nonlinear function $f \in C^{1}(\mathbb{R})$ satisfies

$$
\begin{aligned}
& |f(v)| \leq C|v|^{p},\left|f^{\prime}(v)\right| \leq C|v|^{p-1} \\
& \left|f^{\prime}\left(v_{1}\right)-f^{\prime}\left(v_{2}\right)\right| \leq C\left(\left|v_{1}\right|^{p-2}+\left|v_{2}\right|^{p-2}\right)\left|v_{1}-v_{2}\right|,
\end{aligned}
$$

where $C$ is independent of $v, v_{1}, v_{2}$ [10]. Using (2.30) and (3.13) in (3.12) we have

$$
\begin{aligned}
& \frac{\mathrm{d}}{\mathrm{d} t}\left\{\frac{\alpha}{2}\left\|w_{x x}\right\|^{2}-\left(w_{t}, w_{x x}\right)\right\}-\left\|w_{t x}\right\|^{2}+\alpha\left\|w_{x x}\right\|^{2} \\
& \leq 3 R_{5}^{2} \int_{0}^{1}\left|w \| w_{x x}\right| \mathrm{d} x+\mu C \int_{0}^{1}\left(\left|u_{x}\right|+\left|v_{x}\right|\right)\left|w_{x}\right|\left|w_{x x}\right| \mathrm{d} x
\end{aligned}
$$

Using the Hölder, Young and Sobolev inequalities in right hand side of (3.14) we obtain the estimate

$$
\begin{aligned}
& \frac{\mathrm{d}}{\mathrm{d} t}\left\{\frac{\alpha}{2}\left\|w_{x x}\right\|^{2}-\left(w_{t}, w_{x x}\right)\right\}-\left\|w_{t x}\right\|^{2}+\alpha\left\|w_{x x}\right\|^{2} \\
& \leq 9 R_{5}^{4}\|w\|^{2}+\frac{1}{4}\left\|w_{x x}\right\|^{2}+\mu C S^{2}\left(\left\|u_{x x}\right\|+\left\|v_{x x}\right\|\right)\left\|w_{x x}\right\|^{2}
\end{aligned}
$$

where $S$ is the constant in the Sobolev inequality. Since $u \in H^{2}(0,1) \cap H_{0}^{1}(0,1)$, there exists a positive constant $D$ such that $\left\|u_{x x}\right\| \leq D$. Then we get

$$
\begin{aligned}
& \frac{\mathrm{d}}{\mathrm{d} t}\left\{\frac{\alpha}{2}\left\|w_{x x}\right\|^{2}-\left(w_{t}, w_{x x}\right)\right\}-\left\|w_{t x}\right\|^{2}+\alpha\left\|w_{x x}\right\|^{2} \\
& \leq 9 R_{5}^{4}\|w\|^{2}+\left\{\frac{1}{4}+2 \mu C S^{2} D\right\}\left\|w_{x x}\right\|^{2} .
\end{aligned}
$$


Adding (3.16) to (3.11) and using Poincaré inequality we obtain

$$
\begin{aligned}
& \frac{\mathrm{d}}{\mathrm{d} t} E(t)+(2 \alpha-1)\left\|w_{t x}\right\|^{2}+\alpha\left\|w_{x x}\right\|^{2} \\
& \leq\left\{9 R_{5}^{4}+18 R_{3}^{2}\right\}\|w\|^{2}+\left\{\frac{1}{2 \lambda_{1}}+18 \mu^{2} R_{4}^{4}\right\}\left\|w_{t x}\right\|^{2} \\
& \quad+\left\{\frac{1}{2 \lambda_{1}}+\frac{1}{4}+2 \mu C S^{2} D\right\}\left\|w_{x x}\right\|^{2}
\end{aligned}
$$

where $R_{3}, R_{4}, R_{5}$, are positive constants and

$$
E(t)=\left\|w_{t}\right\|^{2}+\alpha\left\|w_{x}\right\|^{2}+\frac{\alpha}{2}\left\|w_{x x}\right\|^{2}-\left(w_{t}, w_{x x}\right) .
$$

Choosing

$$
\frac{\alpha}{2}=\max \left\{9 R_{5}^{4}+18 R_{3}^{2}, \frac{1}{2 \lambda_{1}}+18 \mu^{2} R_{4}^{4}, \frac{1}{2 \lambda_{1}}+\frac{1}{4}+2 \mu C S^{2} D\right\}
$$

in (3.17) leads to

$$
\begin{aligned}
& \frac{\mathrm{d}}{\mathrm{d} t} E(t)+\left(\frac{3 \alpha}{2}-1\right)\left\|w_{t x}\right\|^{2}+\frac{\alpha}{2}\left\|w_{x x}\right\|^{2} \\
& \leq\left\{9 R_{5}^{4}+18 R_{3}^{2}\right\}\|w\|^{2} .
\end{aligned}
$$

Let $\beta_{\widetilde{I}}$ denote the completeness defect between $\Gamma=H^{2}(0,1) \cap H_{0}^{1}(0,1)$ and $L^{2}(0,1)$ and that is

$$
\begin{aligned}
& \beta_{\mathfrak{I}}\left(\Gamma, L^{2}(0,1)\right) \\
& =\sup \left\{\|w\|: w \in \Gamma, \ell_{j}(w)=0, \ell_{j} \in \mathfrak{J},\|w\|_{\Gamma} \leq 1\right\} .
\end{aligned}
$$

From Theorem 10 we have

$$
\|w\| \leq \beta_{\mathfrak{I}}\left\|w_{x x}\right\|+C_{\mathfrak{I}} \max _{j=1,2, \ldots, n}\left|\ell_{j}(w)\right|
$$

for all $w \in \Gamma$. Squaring both sides of (3.20) and using Cauchy's inequality we obtain

$$
\|w\|^{2} \leq 2 \beta_{\Im}^{2}\left\|w_{x x}\right\|^{2}+2 C_{\Im}^{2} \max _{j=1,2, \ldots, n}\left|\ell_{j}(w)\right|^{2} .
$$

Combining (3.21) in (3.18) leads to

$$
\begin{aligned}
& \frac{\mathrm{d}}{\mathrm{d} t} E(t)+\left(\frac{3 \alpha}{2}-1\right)\left\|w_{t x}\right\|^{2}+\frac{\alpha}{2}\left\|w_{x x}\right\|^{2} \\
& \leq\left\{9 R_{5}^{4}+18 R_{3}^{2}\right\}\left\{2 \beta_{\Im}^{2}\left\|w_{x x}\right\|^{2}+2 C_{\mathfrak{I}}^{2} \max _{j=1,2, \ldots, n}\left|\ell_{j}(w)\right|^{2}\right\}
\end{aligned}
$$

Then we choose $\beta_{\mathfrak{J}}>0$ as small as possible so that $C_{6}=\frac{\alpha}{2}-2 \beta_{\mathfrak{I}}^{2}\left\{9 R_{5}^{4}+18 R_{3}^{2}\right\}>0$. Hence, from (3.22) we have

$$
\begin{aligned}
& \frac{\mathrm{d}}{\mathrm{d} t} E(t)+\left(\frac{3 \alpha}{2}-1\right)\left\|w_{t x}\right\|^{2}+C_{6}\left\|w_{x x}\right\|^{2} \\
& \leq 2\left\{9 R_{5}^{4}+18 R_{3}^{2}\right\} C_{\mathfrak{J}}^{2} \max _{j=1,2, \cdots, n}\left|\ell_{j}(w)\right|^{2}
\end{aligned}
$$

and using Poincaré inequality in (3.23) we find

$$
\begin{aligned}
& \frac{\mathrm{d}}{\mathrm{d} t} E(t)+\lambda_{1}\left(\frac{3 \alpha}{2}-1\right)\left\|w_{t}\right\|^{2}+\frac{C_{6}}{2} \lambda_{1}\left\|w_{x}\right\|^{2}+\frac{C_{6}}{2}\left\|w_{x x}\right\|^{2} \\
& \leq 2\left\{9 R_{5}^{4}+18 R_{3}^{2}\right\} C_{\Im}^{2} \max _{j=1,2, \cdots, n}\left|\ell_{j}(w)\right|^{2}
\end{aligned}
$$

Now we find upper and lower bounds for the functional $E(t)$ owing to the Cauchy-Schwartz and the Cauchy inequalities:

$$
\left|\left(w_{t}, w_{x x}\right)\right| \leq \frac{1}{\alpha}\left\|w_{t}\right\|^{2}+\frac{\alpha}{4}\left\|w_{x x}\right\|^{2} .
$$

Therefore, using (3.25) and from the definition of $E(t)$, we can find that

$$
\begin{aligned}
& \left(1-\frac{1}{\alpha}\right)\left\|w_{t}\right\|^{2}+\left\|w_{x}\right\|^{2}+\frac{\alpha}{4}\left\|w_{x x}\right\|^{2} \\
& \leq E(t) \leq\left(1+\frac{1}{\alpha}\right)\left\|w_{t}\right\|^{2}+\left\|w_{x}\right\|^{2}+\frac{3 \alpha}{4}\left\|w_{x x}\right\|^{2} .
\end{aligned}
$$

Hence, from (3.26) we can obtain that there exists a positive constant

$$
\eta=\min \left\{\frac{\lambda_{1} \alpha(3 \alpha-2)}{2(\alpha+1)}, \frac{C_{6}}{2} \lambda_{1}, \frac{2 C_{6}}{3 \alpha}\right\}
$$

such that

$$
\begin{aligned}
& \frac{\mathrm{d}}{\mathrm{d} t} E(t)+\eta E(t) \\
& \leq 2\left\{9 R_{5}^{4}+18 R_{3}^{2}\right\} C_{\mathfrak{J}}^{2} \max _{j=1,2, \cdots, n}\left|\ell_{j}(w)\right|^{2} .
\end{aligned}
$$

Applying Lemma 11 to (3.27) with

$$
\kappa^{+}(t)=2\left\{9 R_{5}^{4}+18 R_{3}^{2}\right\} C_{\mathfrak{J}}^{2} \max _{j=1,2, \cdots, n}\left|\ell_{j}(w(t))\right|^{2},
$$

$\zeta(t)=\eta$, and $\xi(t)=E(t)$ and using a result of Lemma 11 we see that if

$$
\int_{t}^{t+T} \kappa^{+}(z) \mathrm{d} z
$$

tends to zero as $t \rightarrow \infty$, then $E(t) \rightarrow 0$. Thus we obtain that

$$
\lim _{t \rightarrow \infty}\left\{\left\|w_{t}\right\|^{2}+\left\|w_{x}\right\|^{2}+\left\|w_{x x}\right\|^{2}\right\}=0
$$

or

$$
\lim _{t \rightarrow \infty}\left\{\left\|u_{t}-v_{t}\right\|^{2}+\left\|u_{x}-v_{x}\right\|^{2}+\left\|u_{x x}-v_{x x}\right\|^{2}\right\}=0 .
$$

As a result from Definition 6 , the set $\mathfrak{I}$ defined on $\Gamma$ is a set of determining functionals for (1.1)-(1.3). Therefore we complete the proof of Theorem 12

\section{Acknowledgements}

The author thanks Professor A. Okay Çelebi for valuable 
hints and discussions.

\section{REFERENCES}

[1] F. Chen, B. Guo and P. Wang, "Long Time Behavior of Strongly Damped Nonlinear Wave Equations," Journal of Differential Equations, Vol. 147, No. 2, 1998, pp. 231241. doi:10.1006/jdeq. 1998.3447

[2] N. I. Karachalios and N. M. Staurakakis, "Global Existence and Blow up Results for Some Nonlinear Wave Equations on $\mathbb{R}^{N}$," Advances in Differential Equations, Vol. 6, No. 2, 2001, pp. 155-174.

[3] A. O. Çelebi and D. Uğurlu, "Determining Functionals for the Strongly Damped Nonlinear Wave Equation," Journal of Dynamical Systems and Geometric Theories, Vol. 5, No. 2, 2007, pp. 105-116. doi:10.1080/1726037X.2007.10698530

[4] I. D. Chueshov, "Theory of Functionals That Uniquely Determine Long-Time Dynamics of Infinitive Dimensional Dissipative Systems," Russian Mathematical Surveys, Vol. 53, No. 4, 1998, pp. 1-58. doi:10.1070/RM1998v053n04ABEH000057

[5] I. D. Chueshov and V. K. Kalantarov, "Determining Functionals for Nonlinear Damped Wave Equations," Mate- maticheskaya Fizika, Analiz, Geometriya, Kharkovskii Matematicheskii Zhurnal, Vol. 8, No. 2, 2001, pp. 215227.

[6] B. Cockburn, D. A. Jones and E. S. Titi, "Determining Degrees of Freedom for Nonlinear Dissipative Systems," Comptes Rendus de I'Académie des Sciences Paris Série I Mathématique, Vol. 321, 1995, pp. 563-568.

[7] J. Duan, E. S. Titi and P. Holmes, "Regularity, Approximation and Asymptotic Dynamics for a Generalized Ginzburg-Landou Equation," Nonlinearty, Vol. 6, No. 6, 1993, pp. 915-933. doi:10.1088/0951-7715/6/6/005

[8] D. Henry, "Geometric Theory of Semilinear Parabolic Equations, Lecture Notes in Mathematics," Springer-Verlag, New York, 1981.

[9] P. Massatt, "Limiting Behavior for Strongly Damped Nonlinear Wave Equations," Journal of Differential Equations, Vol. 48, No. 3, 1983, pp. 334-349. doi:10.1016/0022-0396(83)90098-0

[10] H. Takeda and S. Yoshikawa, "On the Initial Value Problem of the Semilinear Beam Equation with Weak Damping II: Asymptotic Profiles," Journal of Differential Equations, Vol. 253, No. 11, 2012, pp. 3061-3080. 